\title{
Demonstration of auroral radio emission mechanisms by laboratory experiment
}

\author{
S L McConville ${ }^{1}$, D C Speirs ${ }^{1}$, K Ronald ${ }^{1}$, A D R Phelps ${ }^{1}$, A W Cross ${ }^{1}$, \\ R Bingham ${ }^{1,3}$, C W Robertson ${ }^{1}$, C G Whyte ${ }^{1}$, W He ${ }^{1}$, K M Gillespie ${ }^{1}$, \\ I Vorgul $^{2}$, R A Cairns ${ }^{2}$ and B J Kellett ${ }^{3}$ \\ ${ }^{1}$ SUPA Department of Physics, University of Strathclyde, G4 0NG, Scotland, UK \\ 2 School of Mathematics and Statistics, University of St. Andrews, KY16 9SS, Scotland, UK \\ ${ }^{3}$ Space Physics Division, STFC, Rutherford Appleton Laboratory, OX11 0QX, England, UK \\ E-mail: sandra.1.mcconville@strath.ac.uk
}

\begin{abstract}
Auroral kilometric radiation occurs in regions of depleted plasma density in the polar magnetosphere. These emissions are close to the electron cyclotron frequency and appear to be connected to the formation of high pitch angle electron populations due to the conservation of the magnetic moment. This results in a horseshoe type distribution function being formed in velocity space where electrons are magnetically compressed as they descend towards the Earth's atmosphere. Satellites have observed that radio emissions occur in conjunction with the formation of this distribution and show the radiation to have propagation and polarization characteristics of the extraordinary (X-mode) plasma mode with emission efficiency observed at $\sim 1-2 \%$. To investigate this phenomenon a laboratory experiment, scaled to microwave frequencies and lab dimensions by increasing the cyclotron frequency, was constructed whereby an electron beam propagated through a region of increasing magnetic field created by five independently variable solenoids. Results are presented for two experimental regimes of resonant coupling, 11.7 and $4.42 \mathrm{GHz}$, achieved by varying the peak magnetic field. Measurements of the experimental radiation frequency, power and efficiency were undertaken as a function of the magnetic compression. Results showed the radiation to be polarized in the near cut-off transverse electric radiation modes, with efficiency of emission $\sim 1-2 \%$, peak power outputs of $\sim 19-30 \mathrm{~kW}$ and frequency close to the cyclotron frequency. This represented close correlation between the laboratory radiation efficiency, spectra, polarization and propagation with that of numerical predictions and the magnetospheric observations.
\end{abstract}




\section{Introduction}

In the auroral region of the Earth's magnetic dipole, satellites have observed horseshoe type distributions in electron velocity space as a result of conservation of the magnetic moment, $\mu$ (equation (1)), by particles subject to magnetic compression as they descend towards the atmosphere. As the magnetic flux density increases, $v_{\perp}$ increases to keep $\mu$ constant, and so $v_{\|}$decreases.

$$
\mu=\frac{m v_{\perp}^{2}}{2 B_{0}},
$$

where $m$ is the electron mass, $v_{\perp}$ is the transverse velocity component and $B_{0}$ is the magnetic flux density.

Auroral kilometric radiation (AKR) (Benediktov et al 1965, Gurnett et al 1974), first observed in 1965 by the satellite 'Electron-2', is the most intense non-thermal natural emission from the Earth, occurring close to the electron cyclotron frequency, $\Omega$ (equation (2)). Similar types of radiation have been observed from the solar system's other magnetized planets Jupiter, Saturn and Uranus (Zarka et al 2001). Recent satellites, including Viking and FAST (Roux et al 1993, Delory et al 1998), have also observed the production of AKR. FAST experimentally measured the horseshoe distribution function with $\left(\partial f / \partial v_{\perp}\right)>0$ in the source region where $f$ is the electron distribution function in velocity space:

$$
\Omega=\frac{e B}{m},
$$

where $e$ is the magnitude of the electron charge and $\Omega$ is the non-relativistic cyclotron frequency.

In 1979, Wu and Lee proposed a mechanism comprising a cyclotron maser instability driven by a positive gradient in the transverse velocity profile of the magnetically mirrored auroral electrons_called the loss cone model (Wu and Lee 1979). In 2000, Bingham and Cairns applied kinetic theory to describe the X-mode dispersion (Bingham and Cairns 2000) and growth due to a cyclotron maser instability driven by a positive gradient in the transverse velocity profile of a horseshoe distribution. This theory accounted for the observed frequencies down to $\omega_{\text {ce }}$ and predicts near perpendicular emission in the X-mode (Benson 1985). New research has considered the spatial variation in the magnetic field as an explanation for the spectral content and temporal stability of AKR emissions (Savilov et al 2007). Equation (3) shows the dispersion relation for a relativistic cyclotron beam. Clearly $k_{\|}$should be as close to 0 as possible for a strong resonance if $v_{\|}$is not well defined (Bratman et al 2000, Chu 2004), consistent with the $\mathrm{X}$-mode. When $k_{\|}$is zero and there is a maximum of $k_{\perp}$ for a given radiation frequency the contours of the resonance are circular in electron velocity space and lie on the inside edge of the horseshoe distribution function. This yields optimal brightness of AKR emission since the radiation can be excited by all components of the electron population (Pritchett and Winglee 1989). As $k_{\|}$increases, the contours of resonance become elliptical in shape and cut across the distribution function. This means the resonance condition is weaker (Vorgul et al 2005).

$$
\omega=S \frac{\Omega}{\gamma}+k_{\|} v_{\|}
$$

where $k_{\|}$is the parallel component of $k$ vector, $v_{\|}$is the parallel component of velocity, $S$ is the cyclotron harmonic number and $\gamma$ is the Lorentz factor.

The horseshoe distributions were shown by kinetic analysis (Bingham and Cairns 2000, Vorgul et al 2005) to be unstable to an electron cyclotron resonance maser type of instability (Twiss et al 1958, Sprangle and Drobot 1977), and are now proposed as the source of free 

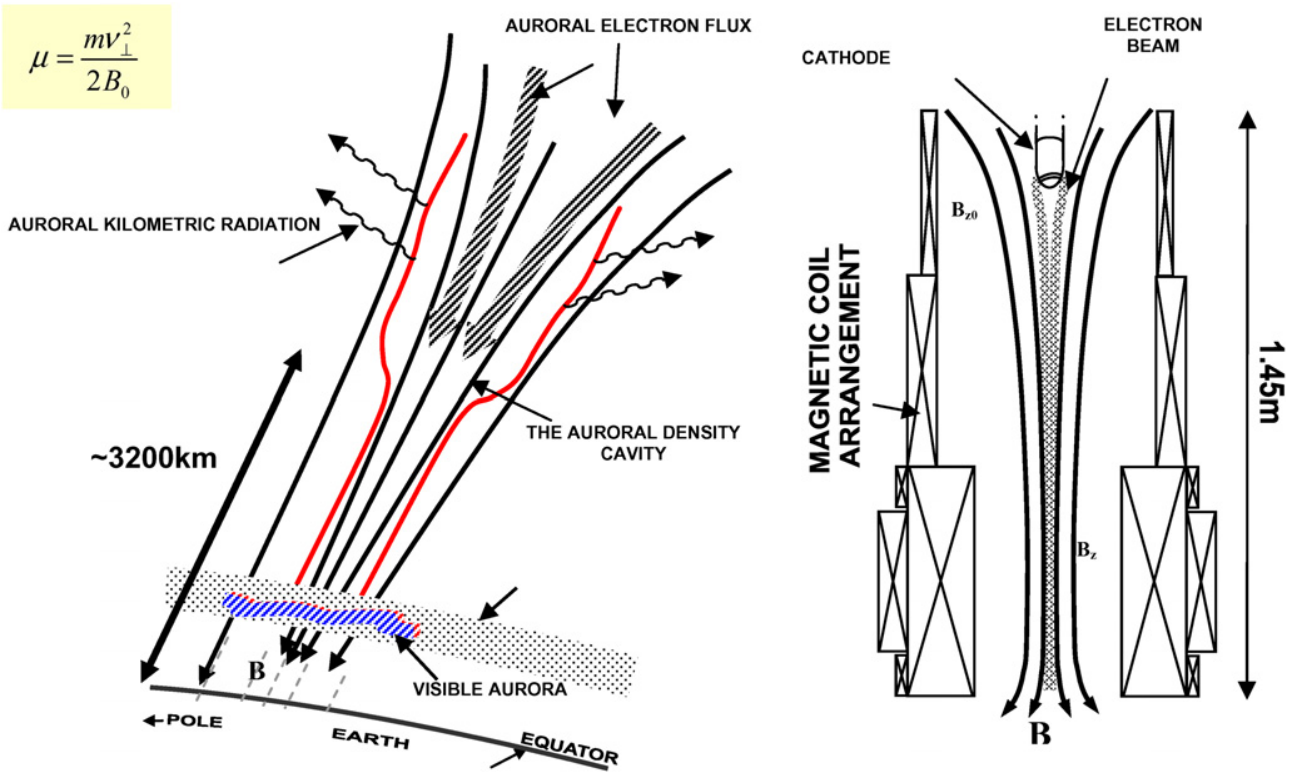

Figure 1. Comparative geometry of the auroral zone and the laboratory experiment.

energy responsible for the production of AKR (Bingham and Cairns 2002, Bingham et al 2004) and other astrophysical radio emissions (Bingham et al 2003, Kellett et al 2002). The electron population in the horseshoe distribution formed through magnetic compression has a high fraction of gyrational momentum allowing energy extraction to be feasible. Figure 1 depicts the auroral zone where the electrons transit the auroral density cavity, a region of plasma depletion. In this region, due to the absence of background plasma, electrons have $\omega_{\mathrm{p}}<\omega_{\mathrm{w}}$, where $\omega_{\mathrm{p}}$ is the electron plasma frequency and $\omega_{\mathrm{w}} \cong \Omega / \gamma$ is the frequency of the wave. Towards the edges of this region $\omega_{\mathrm{p}}$ increases markedly thus creating a boundary effect (Burinskaya and Rauch 2007). Satellites that investigated these auroral regions have observed that the radiation is emitted in the extraordinary (X) plasma mode (Ergun et al 1998, Ergun et al 2000), polarized and propagating perpendicular to the static magnetic field. More distant observations indicate some small fraction is also polarized in the ordinary $(\mathrm{O})$ mode (Hanasz et al 2003). In the magnetosphere a parallel electric field, $E_{\|}$, is necessary to constantly accelerate the electrons into the converging magnetic field to obtain the AKR distribution (Wu and Lee 1979, Pritchett and Winglee 1989) and reduce the plasma density.

To reproduce major features of this auroral situation, a laboratory experiment was constructed, scaled from lengths $\sim 9000 \mathrm{~km}$, the extent of the density cavity, down to $\sim 2 \mathrm{~m}$ and from frequencies of $\sim 300 \mathrm{kHz}$ to between 4 and $12 \mathrm{GHz}$. As this experiment intended to study the generation efficiency from directly within the source region the authors have concentrated on resonances with X-like modes. The role of the parallel electric field was provided by an electron gun which injected an electron beam with an initial velocity spread into a set of electromagnets where it was subject to magnetic compression. This gave the effect of the auroral density cavity with the waveguide walls acting as the boundary, whilst the solenoids provided the 'bottleneck' of increasing magnetic field. Measurements were undertaken of the electron beam's current and energy. The results showed the formation of the desired horseshoe shape in the electron beams velocity distribution, reproducing the magnetospheric situation. Measurements of the output power, emission efficiency, operating frequency, emitted wave 


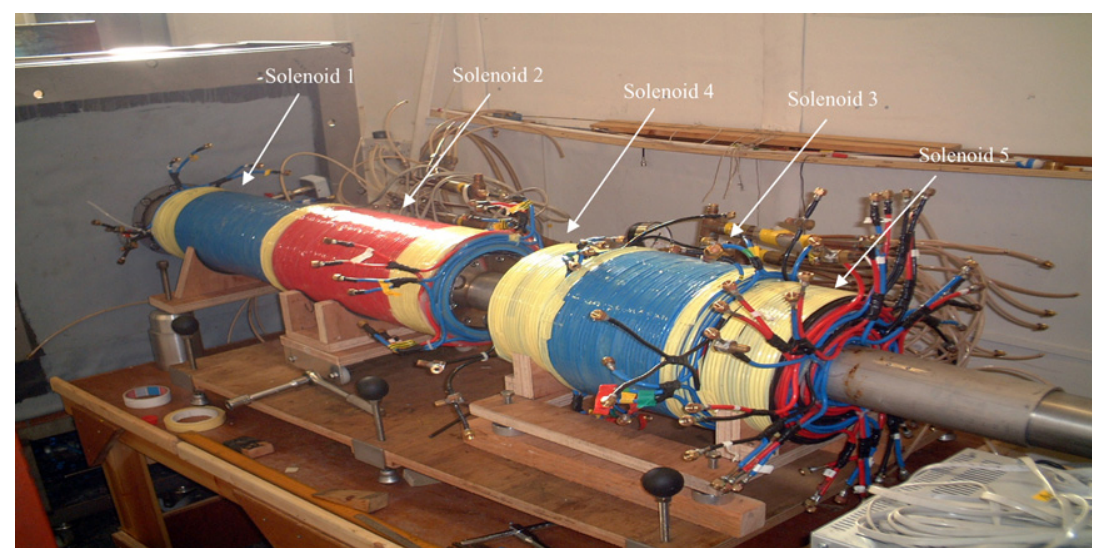

Figure 2. The solenoids of the magnetic field system during assembly in the laboratory.

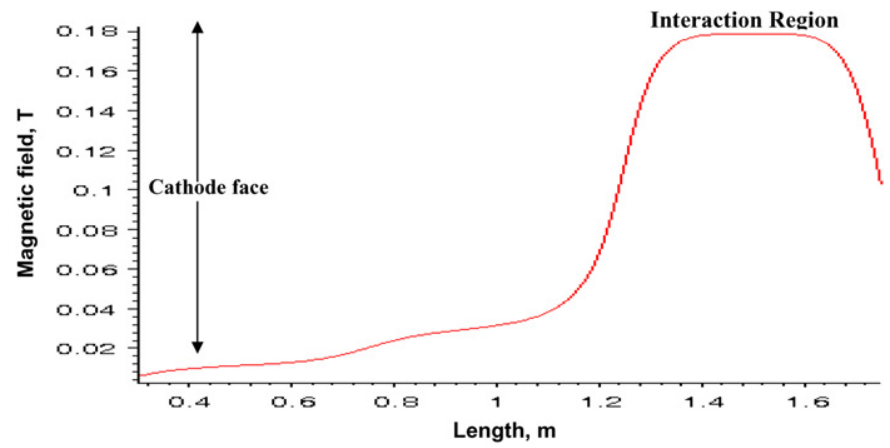

Figure 3. Magnetic field profile through the centre of the solenoids. The locations of the cathode and interaction region are marked. This profile was calculated by computer and checked experimentally by Hall sensor.

polarization and propagation were taken as a function of detuning between the radiation and beam frequencies and degree of magnetic compression. These were compared with numerical and theoretical predictions (Cairns et al 2005, Speirs et al 2005, Vorgul et al 2005 and Speirs et al 2008) and the magnetospheric observations.

\section{Experimental apparatus}

A system of solenoids was created as shown in figure 2, to encompass the electron beam as it traversed through the experiment from the cathode through the interaction space to the anode. Each of the five solenoids was constructed by winding OFHC copper tubing around non-magnetic formers. The individual copper tubes were around $20 \mathrm{~m}$ in length with an ID of $2.5 \mathrm{~mm}$ and $\mathrm{OD}$ of $7 \mathrm{~mm}$, coated in dielectric insulation. A high pressure water cooling system, delivering a coolant inlet pressure of $\sim 22$ bar to the tubing core, allowed the solenoids to be run up to a power of $120 \mathrm{~kW}$ with up to $300 \mathrm{~A}$ on each individual wire. Independent adjustment of each of the coils allowed fine control of the magnetic field compression profile, figure 3 . The electron injector was placed in the low, fringing, magnetic field generated by solenoid 1 which ensured that the electrons had an initial spread in their pitch angles. The electrons 


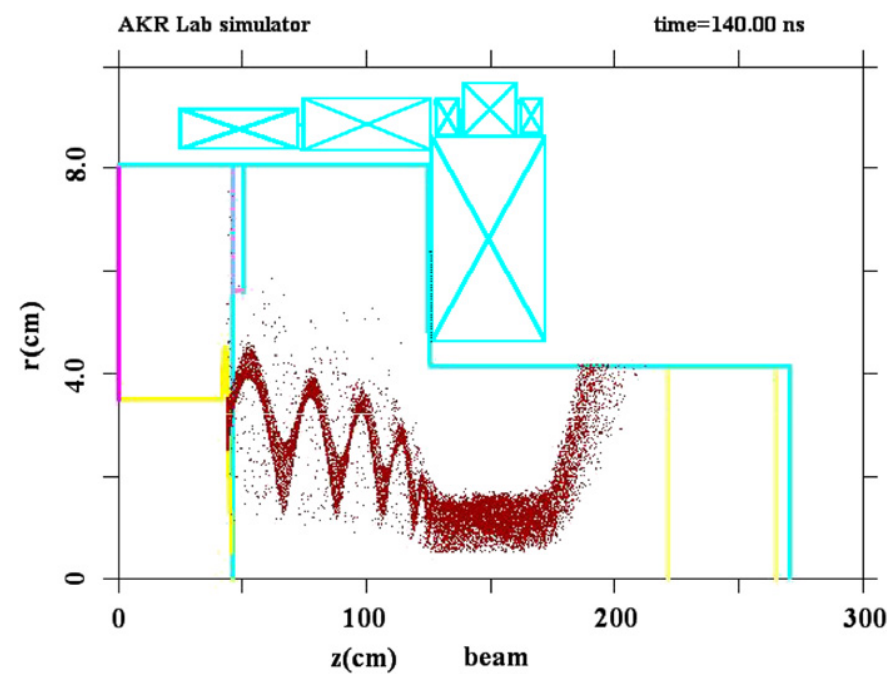

Figure 4. Electron beam trajectory through the apparatus as predicted by the PiC code KARAT.

were subject to magnetic compression as they passed into solenoid 2 and reached a maximum magnetic field in the plateau in the centre of solenoid 3. The shimming solenoids 4 and 5 were used to balance the profile of the magnetic field plateau. Two different plateau magnetic fields were used. For the low frequency resonance experiment at $4.42 \mathrm{GHz}$ the flux density of the plateau was $0.18 \mathrm{~T}$. For the higher frequency resonance of $11.7 \mathrm{GHz}$ the flux density was $0.49 \mathrm{~T}$ (Speirs et al 2005). The solenoids determined the size of the evacuated anode vacuum vessel/beam tunnel and interaction space $16 \mathrm{~cm}$ and $8.28 \mathrm{~cm}$ in diameter, respectively, illustrated in figure 4, which also shows the computed electron trajectories as the beam travels through the system. The beam tunnel is the area between the mesh and the entrance to the interaction region. At the walls where the beam tunnel leads into the interaction region there is a slope in their shape; this is to allow as much of the beam as possible into the $8.28 \mathrm{~cm}$ diameter waveguide. It was essential that the system be kept under vacuum, $\sim 10^{-6}$ mbar, so that there were no unnecessary collisions between the electrons and any other particles in the residual gas.

The electron gun used in the system had a vacuum spark cathode; these types of devices are pulsed cathodes used to generate a high voltage, short duration electron beam. The cathode used in the present experiment is energized by $100 \mathrm{~ns}$ pulses from a Blumlein power supply (Somerville et al 1990) capable of reaching up to $200 \mathrm{kV}$. The output of the quad cable Blumlein system, figure 5(a), was matched by an ionic resistor connecting its high voltage terminal to an earth connection. The Blumlein cables were charged in parallel and discharged in series when a triggered mid-plane spark gap reached breakdown to give an amplified output signal (up to $3.5 \times V_{\mathrm{ch}}$ ) whilst maintaining a rectangular output pulse. The ionic resistor that is connected to the high voltage end of the Blumlein is made up of a saturated copper sulphate $\left(\mathrm{CuSO}_{4}\right)$ solution, which has the advantage of being able to withstand high peak powers without damage. The role of the matching resistor, $\sim 230 \Omega$, was to match the impedance of the Blumlein, $200 \Omega$, so that the signal did not reflect back to the supply when the experiment was energized. If the matching resistor were not present, the power from the initial pulse would reflect back into the supply and cause undesirable amplitude modulation.

The length of the cathode stalk and the placement of the anode mesh were adjusted to set a diode gap of $2 \mathrm{~cm}$. From figure 5(b), the face of the cathode can be seen with the velvet ring 


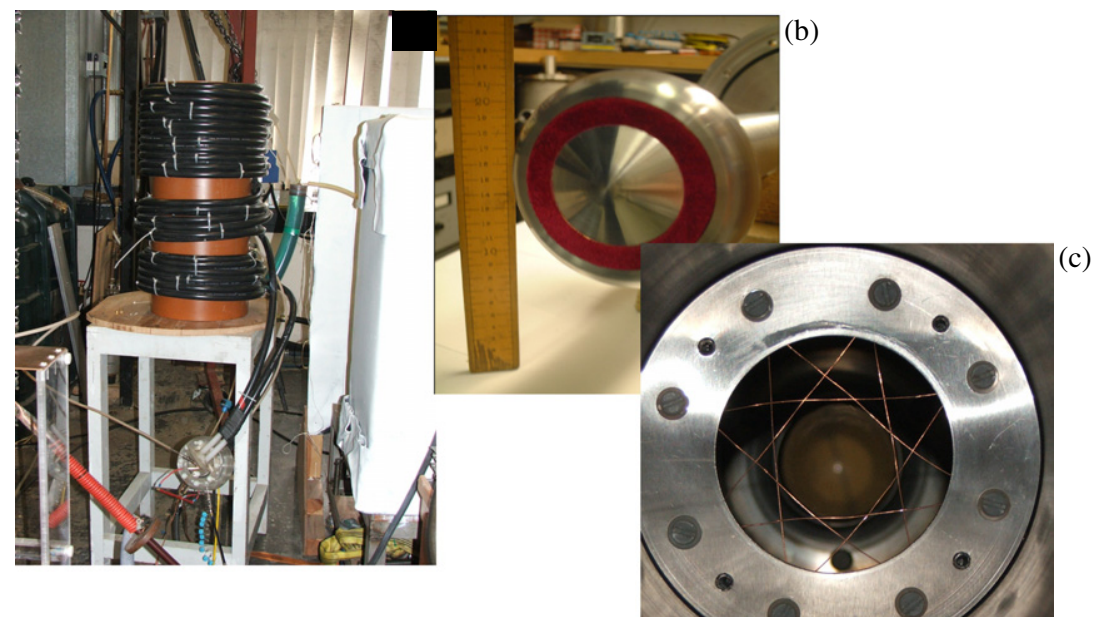

Figure 5. (a) Blumlein pulsed power supply, (b) cathode face with velvet ring, $(c)$ anode mesh.

surrounding the dome-shaped cathode surface. When the output pulse from the power supply energized the cathode, electrons were emitted due to field emission from the velvet fibres. As the temperature at the fibre tips increased, the field emission led to the formation of a plasma cloud and ultimately resulted in explosive electron emission. The rate at which the plasma travelled across the cathode-mesh gap was determined by the material used (Mesyats and Proskurovsky 1989, Ronald et al 1998, Mesyats 2005): for velvet the expansion velocity is of the order $2 \mathrm{~cm} \mu \mathrm{s}^{-1}$. An expansion of a few centimetres per microseconds limits the minimum gap spacing to a couple of centimetres for pulses of $100 \mathrm{~ns}$. The shape of the cathode face was designed to make those electrons on the inner circumference of the velvet emission ring have more transverse momentum than those on the outer circumference to induce a moderate spread in the velocity of the electron beam. The anode mesh, figure 5(c), had a sparse grid and was used to set the anode-cathode gap to provide a sufficiently high field $\sim 50 \mathrm{kV} \mathrm{cm}^{-1}$ on the cathode to cause ignition of the plasma flare. A sparse mesh was used to avoid excessive loss of high pitch factor electrons which would arise if small aperture holes were employed. The electrons were brought to cyclotron resonance in the $8.28 \mathrm{~cm}$ diameter tube with near cut-off transverse electric (TE) modes as these share similar polarization and propagation properties (ac electric field and the wave vector are both perpendicular to the static magnetic field) with the X-mode observed in the magnetosphere. In particular resonances with $\mathrm{TE}_{(0, n)}$ modes were studied as these could be modelled in $2 \mathrm{D} \mathrm{PiC}$ code simulations due to their azimuthal symmetry (Speirs et al 2008). Equation (3) must be satisfied for resonant interaction and energy exchange to occur. To excite the near cut-off $\mathrm{TE}_{01}$ mode at a frequency of $4.42 \mathrm{GHz}$, the plateau magnetic flux density was set at $0.18 \mathrm{~T}$, the low frequency resonance regime. The same analysis determined the higher frequency resonance regime of $11.7 \mathrm{GHz}$ for the $\mathrm{TE}_{03}$ mode requiring a plateau flux density of $0.49 \mathrm{~T}$ (Ronald et al 2008).

\section{Diagnostics}

Accelerating potential was measured by sensing the current through the matching ionic resistor by a Rogowski belt and recorded by a deep memory oscilloscope. Diode current was obtained with a Rogowski belt diagnostic placed in the ground current return connection of the anode. 
The beam current was measured by placing a Faraday cup beam interceptor into the interaction space. The Faraday cup was conical in shape to inhibit the escape of any secondary electrons that were produced.

Pairs of waveguide 12 and waveguide 18 stub receiver systems were created. These devices were built using single mode linearly polarized rectangular waveguide components with simple open ended waveguide apertures for $4.42 \mathrm{GHz}$ and $11.7 \mathrm{GHz}$ radiation, respectively. These were fitted with calibrated attenuators, placed in the far field of the experiment output antenna and fed signals to calibrated rectifying diodes. The rectified microwave radiation pulses were recorded using a $1.5 \mathrm{GHz}$ deep memory digital oscilloscope. To measure the microwave spectra a deep memory $12 \mathrm{GHz}$ digital oscilloscope was used to capture the radiation ac waveforms. Fourier transform of these allowed the radiation frequency to be measured. At the higher resonance regime, it was necessary to use cut-off filters to confirm the spectrum since the frequency was close to the oscilloscope's bandwidth limit. The pairs of waveguide stub antennae were used to measure the radiation antenna pattern emitted by the experiment in radial and azimuthal polarizations as a function of the azimuthal angle. One antenna was used to scan in the azimuthal direction and one provided a control reference. These readings were then analysed to give the power output of the system (Ronald et al 2008). Measurements were taken of azimuthal angles of up to $55^{\circ}$, angles greater than $55^{\circ}$ could not be measured due to the walls of the output flange obstructing the radiation emission. Two regimes of resonant interaction were investigated, one at $4.42 \mathrm{GHz}$ and the second at $11.7 \mathrm{GHz}$. For the lower frequency, a maximum magnetic flux density plateau of $0.18 \mathrm{~T}$ was set in the interaction region of solenoid 3. This gave resonance near cut-off with a relatively low order mode, $\mathrm{TE}_{01}$, whilst retaining a substantial reserve capacity of magnetic field with which the resonant electron distribution function was measured. The higher frequency was investigated to more closely replicate the magnetospheric situation with $\lambda \ll$ dimensions of the resonant cavity (Burinskaya and Rauch 2007). This frequency required a maximum plateau magnetic field of $0.49 \mathrm{~T}$.

\section{Experimental results}

The beam energy, current and transport through the apparatus were investigated as a function of the configuration of the magnetic field. Examples of the beam current and diode voltage traces observed on the oscilloscope are presented in figure 6(a). At (i) on the diagram the output pulse from the Blumlein reached its maximum amplitude. When the cathode was energized by this pulse it released electrons due to field emission which resulted in explosive electron emission (Ronald et al 1998, Mesyats 2005) and the onset of a vacuum spark in the electron gun. The experiment impedance at this point reduced sharply reducing the total load impedance seen by the Blumlein and the voltage across the diode to $75 \mathrm{kV}$, (ii) on the diagram. Figure $6(b)$ indicates how the beam current pulse varied as a function of the gun magnetic field for a beam voltage of $85 \mathrm{kV}$, plateau flux density of $0.52 \mathrm{~T}$, and shows that as the cathode flux density was increased from 0.013 to $0.05 \mathrm{~T}$, the beam current increased from 12 to $47 \mathrm{~A}$. Figure $6(c)$ displays similar information but with a lower beam voltage of $75 \mathrm{kV}$, plateau flux density of $0.4 \mathrm{~T}$ and shows that as the cathode flux density was increased from 0.01 to $0.05 \mathrm{~T}$, the current of the electron beam increased from 6 to $47 \mathrm{~A}$. Results for the fraction of beam transported into the interaction region for a given cathode flux density as the maximum plateau magnetic field was increased, demonstrated the formation of the horseshoe distribution, because progressive magnetic mirroring was achieved. Analysing the variation of the beam current with mirror ratio, figure 7(a), as the plateau magnetic field was increased from the resonant value and relating this back to the critical value of $v_{z}$ yielded the one-dimensional (1D) number density 

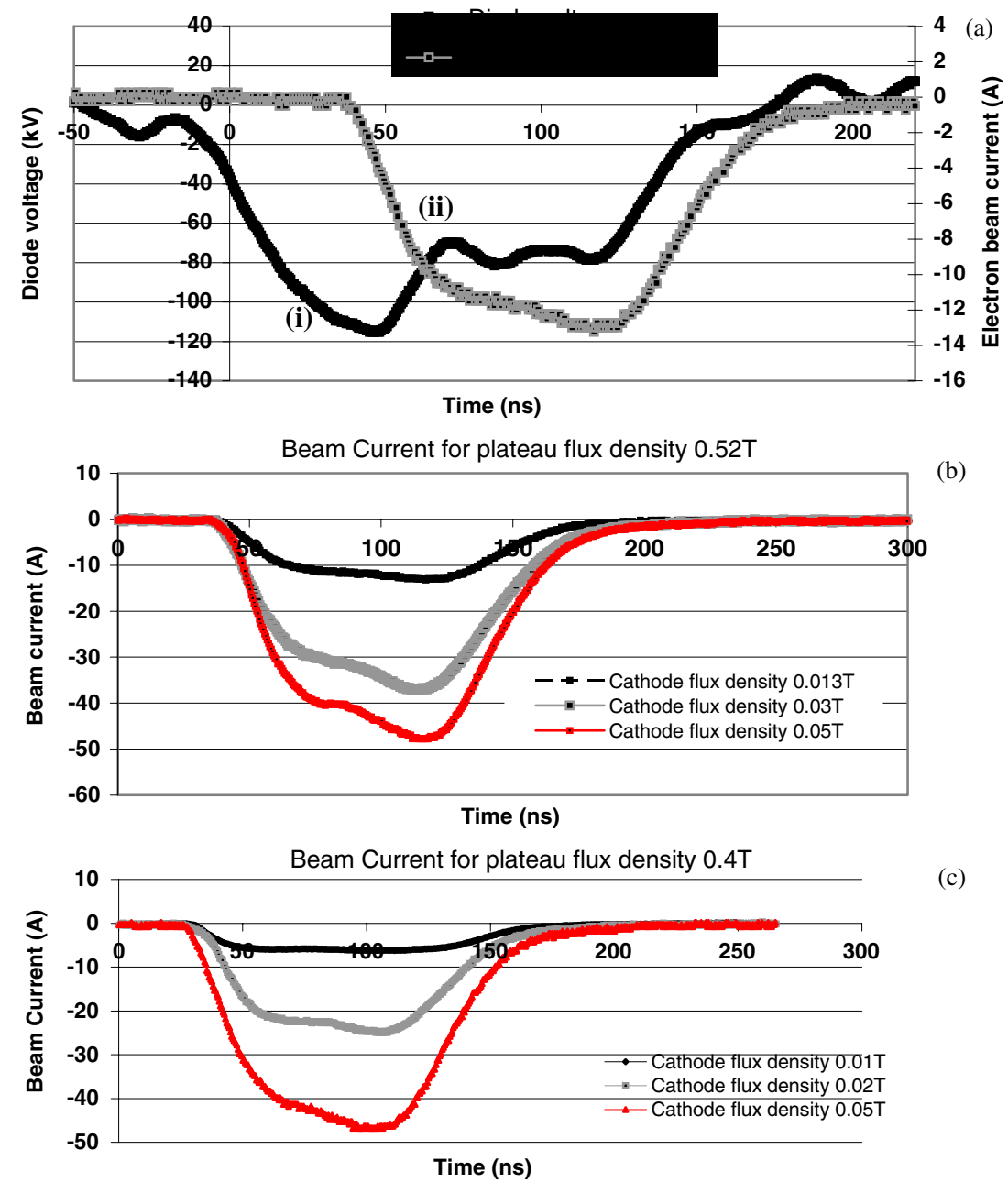

(c)

Figure 6. (a) Raw diode voltage and beam current plot, $(b)$ beam current at plateau flux $0.52 \mathrm{~T}$, (c) beam current at plateau flux $0.4 \mathrm{~T}$.

of the electron beam as a function of the pitch angle. The 1D number density of the electron beam formed at the cathode flux densities of 0.01 and $0.02 \mathrm{~T}$ and plateau flux density of $0.18 \mathrm{~T}$ can be seen as a function of pitch angle in figure 7(b).

Analysis of figure 7 demonstrates that for the lower cathode flux density of $0.01 \mathrm{~T}$, the electron beam had half the number density at high pitch angles compared with the flux density of $0.02 \mathrm{~T}$. For the $0.01 \mathrm{~T}$ case, $2 / 3$ of the current was associated with electrons having pitch angles greater than $37^{\circ}$. Electrons having a pitch $<37^{\circ}$ were not measured due to apparatus limitations. For the $0.02 \mathrm{~T}$ case, only $1 / 3$ of the current was associated with electrons having pitch angles $>37^{\circ}$.

Figure 8(a) shows the radiation ac waveform for the lower resonance regime of $4.42 \mathrm{GHz}$ from which the Fourier transform was calculated, figure $8(b)$. The peak spectral component of the signal was observed at $4.42 \mathrm{GHz}$ close to cut-off for the $\mathrm{TE}_{01}$ mode and the cyclotron frequency, with a second harmonic observed at $\sim 8.8 \mathrm{GHz}$. The maximum efficiency was achieved at a cyclotron detuning $\left(\omega_{\mathrm{w}}-\omega_{\mathrm{ce}}\right) / \omega_{\mathrm{ce}}$ of $\sim 2.5 \%$. Figure 9 shows the experimental 


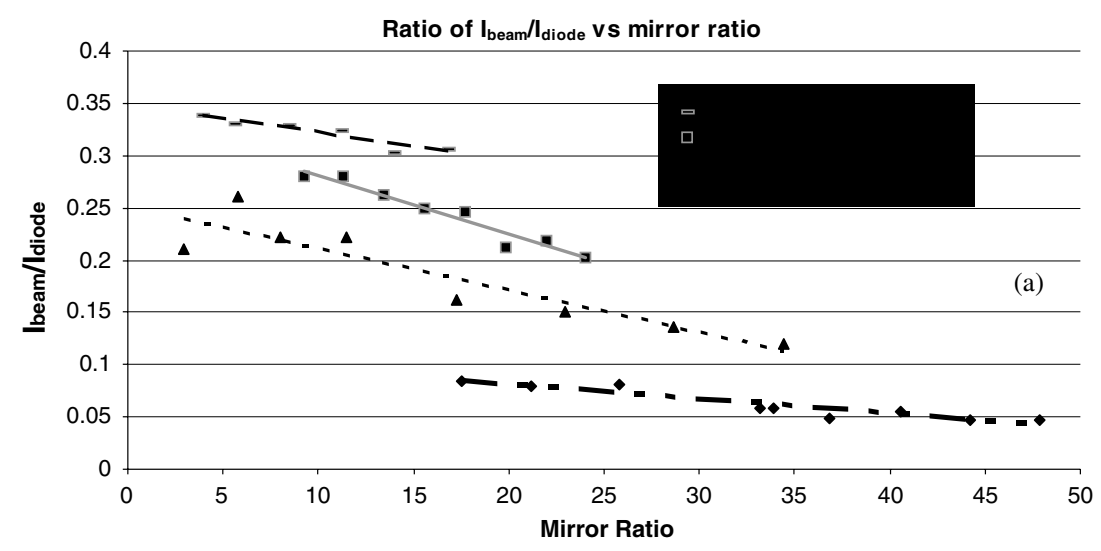

1D Number Density Distribution vs Pitch Angle with Plateau

Flux Density $0.18 T$

- Cathode flux density $0.01 \mathrm{~T}, \mathrm{Op} \mathrm{I}=12 \mathrm{~A}$

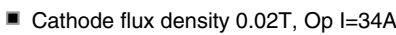

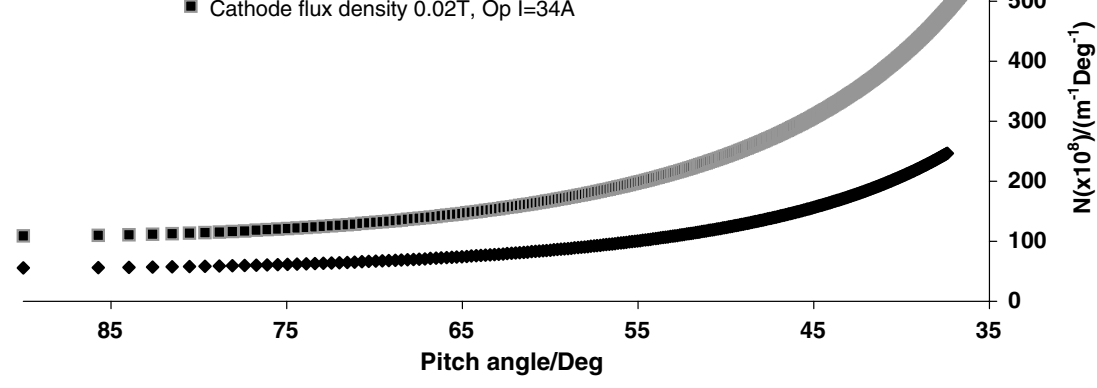

Figure 7. (a) Variation of electron beam transport from accelerator to interaction space as a function of the magnetic configuration, $(b)$ analysis of the 1D number density distribution at a plateau flux density of $0.18 \mathrm{~T}$.

and normalized theoretical microwave antenna pattern measurements. The two plots are in close agreement up to an angle of $\sim 45^{\circ}$, confirming the mode to be the $\mathrm{TE}_{01}$ at $4.42 \mathrm{GHz}$. Excitation of a near cut-off TE mode was consistent with AKR, which is predominately generated in the X-mode propagating and polarized perpendicular to the static magnetic field. The discrepancy above $45^{\circ}$ was due to scattering of the experimental pattern from part of the vacuum window. Integration of the normalized theoretical antenna pattern obtained from these mode scan measurements gave output powers of $19 \mathrm{~kW}$ and $35 \mathrm{~kW}$ for cathode flux densities of $0.01 \mathrm{~T}$ and $0.02 \mathrm{~T}$, with maximum efficiencies of emission of $2 \%$ and $1 \%$, respectively. The higher power but lower efficiency associated with the $0.02 \mathrm{~T}$ regime is consistent with the distribution of the particles noted in the 1D number density plot, figure 7(b). Figure 10(a) shows the Fourier transform for the higher resonance regime where a peak signal was observed at $11.7 \mathrm{GHz}$, close to the cyclotron frequency and the cut-off for the $\mathrm{TE}_{03}$ mode. As the resonance in this case was close to the limit of the oscilloscope, cut-off filters were used in the waveguide to confirm that no significant higher spectral components were present, figure $10(b)$. The antenna patterns for the higher resonance regime of $11.7 \mathrm{GHz}$ can be seen in figure 11. Due to the multimode nature of this resonance condition, the measured mode plot was more complicated and the energy was split between the azimuthal and radial polarizations. Components of both the $\mathrm{TE}_{03}$ and $\mathrm{TE}_{23}$ modes were observed, and the experiment appeared to exhibit mode hopping where the dominant resonant mode changed with time and there were 

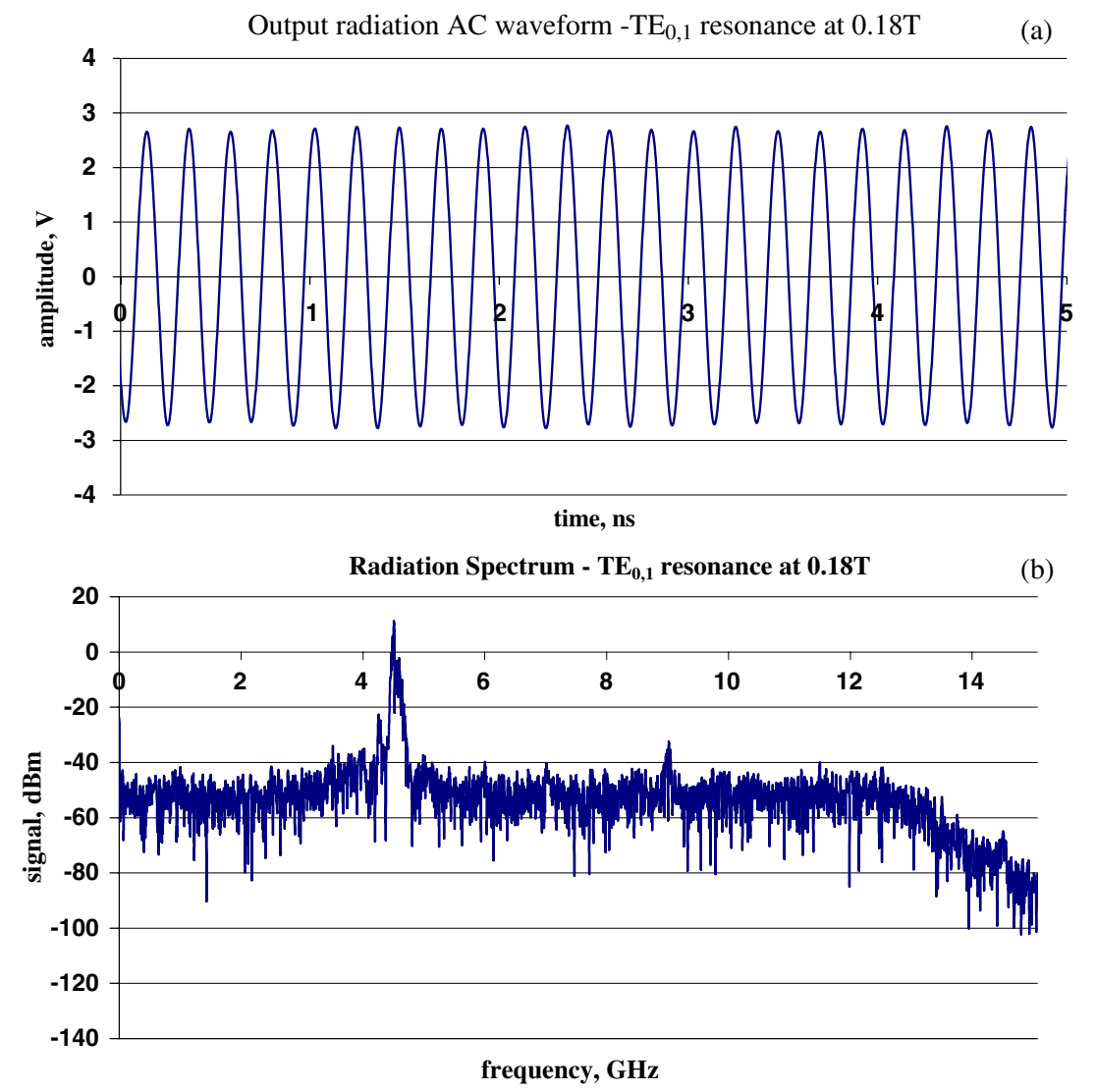

Figure 8. (a) Ac waveform for $4.42 \mathrm{GHz}$ microwave results, (b) Fourier transform with frequency peak at $4.42 \mathrm{GHz}$.

two temporally separated peaks in the output radiation. At a cathode flux density of $0.013 \mathrm{~T}$, power of up to $9.4 \mathrm{~kW}$ was observed and at a cathode flux density of $0.03 \mathrm{~T}$ a power of up to $30 \mathrm{~kW}$ was emitted. A maximum efficiency of emission was achieved with a cathode flux density of $0.03 \mathrm{~T}$, beam current $37 \mathrm{~A}$ of $\sim 1 \%$.

\section{Summary}

A laboratory experiment was constructed using electromagnets to produce a progressively increasing magnetic field profile, through which an electron beam was transported to create a horseshoe distribution function in electron velocity space. Microwave radiation was extracted by the postulated AKR mechanism. The production of the horseshoe electron distribution function, at a maximum magnetic field corresponding to a cyclotron frequency of $4.42 \mathrm{GHz}$ was shown by the beam transport measurements. Microwave measurements confirmed the instability of the distribution function to cyclotron emissions, whilst frequency measurements showed the radiation frequency to be just above the relativistic cyclotron frequency. Integration of the antenna patterns gave a maximum efficiency of up to $2 \%$ agreeing with both numerical predictions and magnetospheric observations. The relationship between the wave and cyclotron frequencies and the wave polarization and propagation directions were also consistent with the satellite measurements. 


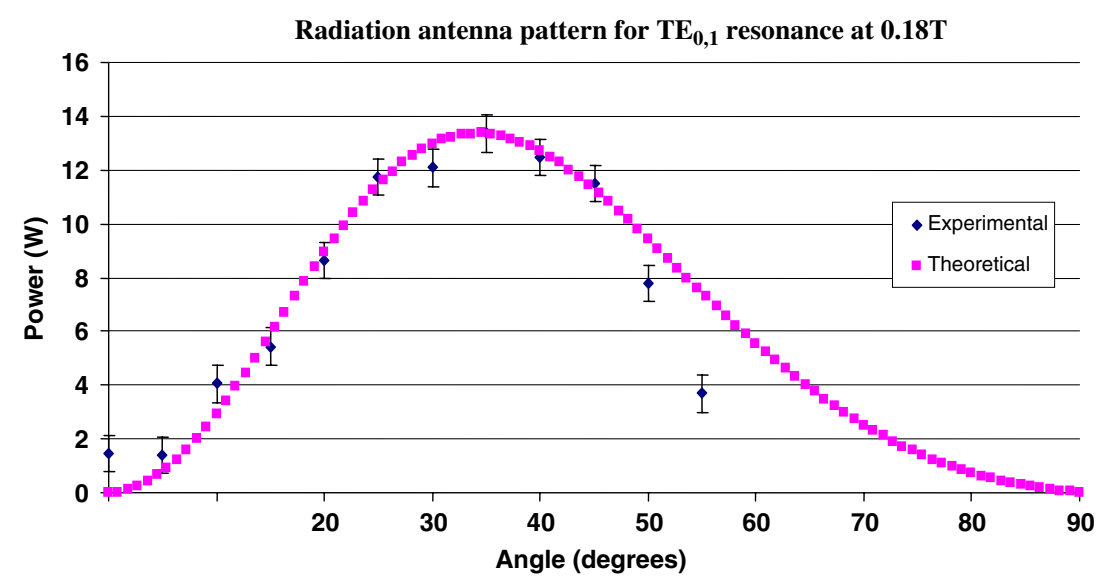

Figure 9. Measurement of the antenna pattern showing the $\mathrm{TE}_{0,1}$ mode.
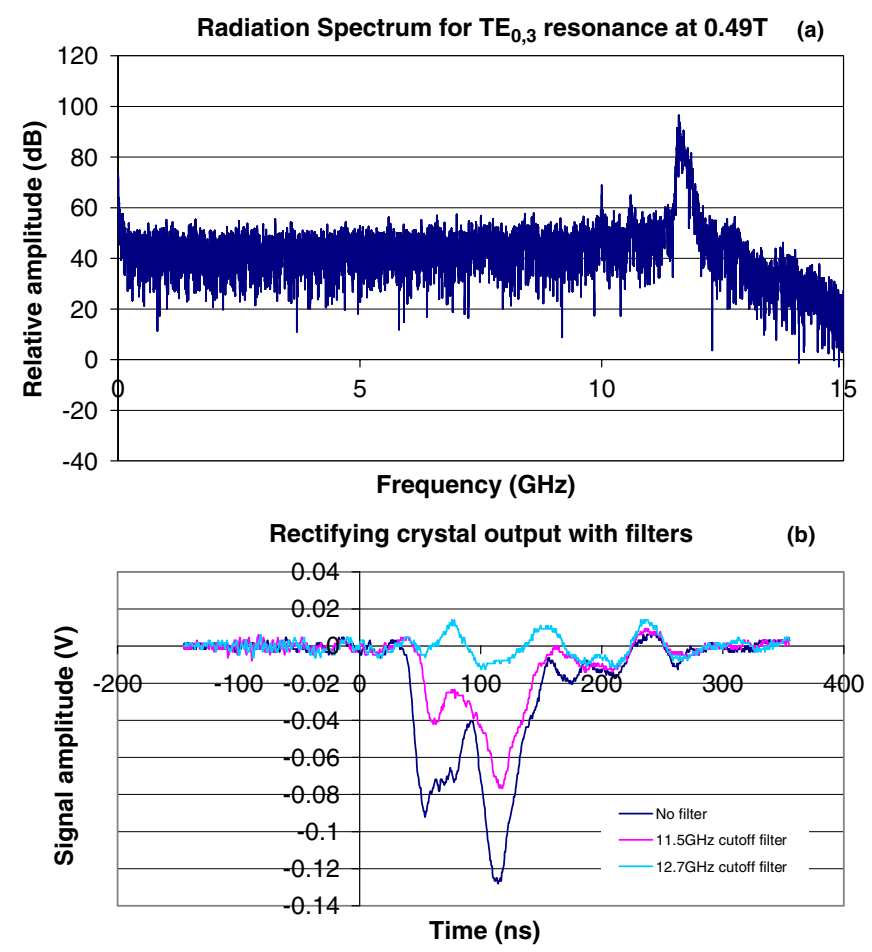

Figure 10. (a) Fourier transform with frequency peak at $11.7 \mathrm{GHz},(b)$ rectifying crystal output with different cut-off filters.

\section{Future work}

To enhance the comparability between the laboratory and magnetospheric geometry, the addition of a background plasma is planned. The proposed scheme will create a Penning gas discharge in the interaction region to form a low temperature, low density ionized gas. This geometry will take advantage of the inherent magnetic field present in the apparatus whilst 

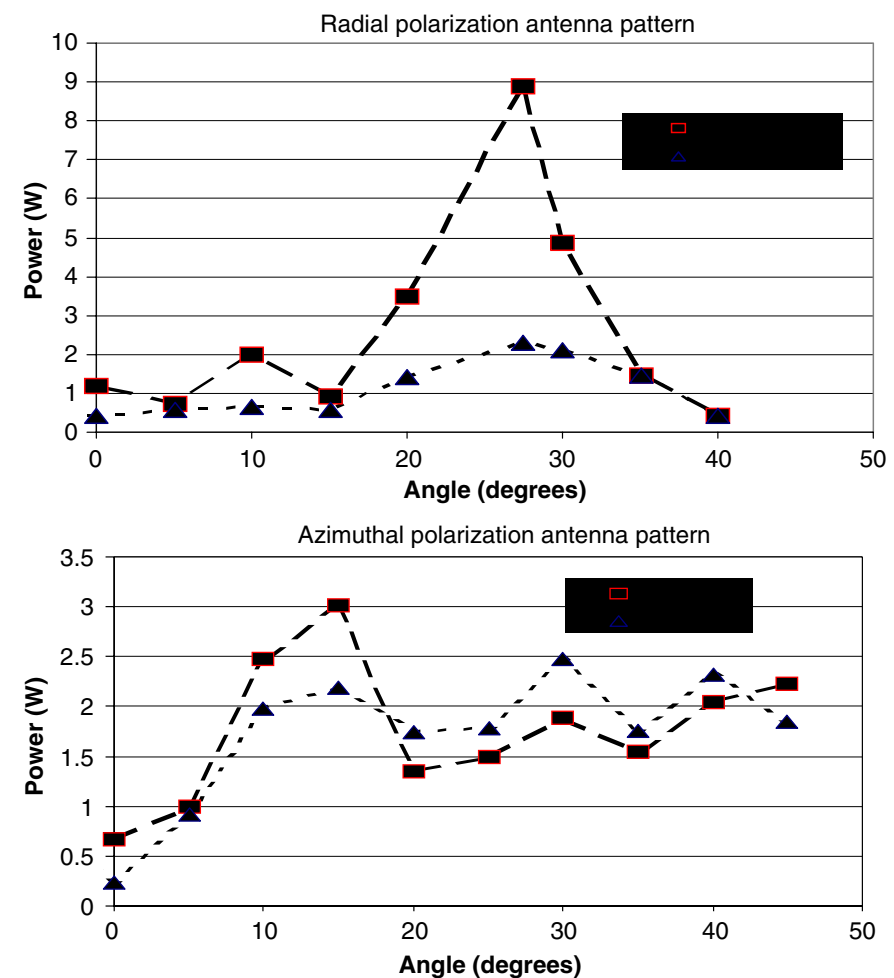

Figure 11. Radial and azimuthal antenna patterns for a resonance at $0.49 \mathrm{~T}$ plateau flux density showing components of the $\mathrm{TE}_{03}$ and $\mathrm{TE}_{23}$ modes and temporal mode hopping.

improving discharge ignition in the low pressure regimes. The addition of the background of 'cool' particles will allow investigation of the role of the weak background plasma in the auroral density cavities, specifically the possibility of upper hybrid resonant absorption.

\section{Acknowledgments}

The EPSRC and the STFC Centre for Fundamental Physics supported this work. Mr I S Dinwoodie is thanked for his help in creating the apparatus and Professor V L Tarakanov for assistance with the numerical model. Helpful conversations with Professor A Savilov are gratefully acknowledged.

\section{References}

Benediktov E A, Getmanstev G G, Sazonov Yu A and Tarasov A F 1965 Cosm. Res. 3 614-7

Benson R F 1985 J. Geophys. Res.-Space Phys. 90 2753-84

Bingham R and Cairns R A 2000 Phys. Plasmas 7 3089-92

Bingham R and Cairns R A 2002 Phys. Scr. T 98 160-2

Bingham R, Kellett B J, Cairns R A, Tonge J and Mendonca J T 2003 Astrophysical J. 595 279-84

Bingham R, Kellett B J, Cairns R A, Vorgul I, Phelps A D R, Ronald K and Speirs D 2004 Contrib. Plasma Phys. $44382-7$

Bratman V L, Cross A W, Denisov G G, He W, Phelps A D, Ronald K, Samsonov S V, Whyte C G and Young A R 2000 Phys. Rev. Lett. $842746-9$ 
Burinskaya T M and Rauch J L 2007 Plasma Phys. Rep. 33 28-37

Cairns R A, Speirs D C, Ronald K, Vorgul I, Kellett B J, Phelps A D R and Bingham R 2005 Phys. Scr. T $11623-6$ Chu K R 2004 Rev. Mod. Phys. 76 489-540

Delory G T, Ergun R E, Carlson C W, Muschietti L, Chaston C C, Peria W, McFadden J P and Strangeway R 1998 Geophys. Res. Lett. 25 2069-72

Ergun R E et al 1998 Geophys. Res. Lett. 25 2061-4

Ergun R E, Carlson C W, McFadden J P, Delory G T, Strangeway R J and Pritchett P L 2000 Astrophys. J. 538 456-66

Gurnett D A 1974 J. Geophys. Res. 79 4227-38

Hanasz J, Panchenko M, de Faeraudy H, Schreiber R and Mogilevsky M M 2003 J. Geophys. Res. 1081408

Kellett B J, Bingham R, Cairns R A and Tsikoudi V 2002 Mon. Not. R. Astron. Soc. 329 102-8

Mesyats G A 2005 Plasma Phys. Control. Fusion 47 109-51

Mesyats G A and Proskurovsky D I 1989 Pulsed Electrical Discharge in Vacuum (Berlin: Springer)

Pritchett P L and Winglee R M 1989 J. Geophys. Res. 94 129-43

Ronald K, Cross A W, Phelps A D R, He W L, Yin H B and Spark S N 1998 IEEE Trans. Plasma Sci. 26 $375-82$

Ronald K, Speirs D C, McConville S L, Phelps A D R, Robertson C W, Whyte C G, He W, Gillespie K M, Cross A W and Bingham R 2008 Radio frequency resonator structure and diagnostic measurements for a laboratory simulation of Auroral Kilometric Radiation Phys. Plasmas 15 at press

Roux A, Hilgers A, de Feraudy H, le Queau D, Louard P, Perraut S, Bahnsen A, Jespersen M, Ungstrup E and Andre M 1993 J. Geophys. Res. $9811657-70$

Savilov A V, Bespalov P A, Ronald K and Phelps A D R 2007 Phys. Plasmas 14113104

Somerville I C, MacGregor S J and Farish O 1990 Meas. Sci. Technol. $1865-8$

Speirs D C, Vorgul I, Ronald K, Bingham R, Cairns R A, Phelps A D R, Kellett B J, Cross A W, Whyte C G and Robertson C 2005 J. Plasma Phys. 71 665-74

Speirs D C et al 2008 Plasma Phys. Control. Fusion 50074011

Sprangle P and Drobot A T 1977 IEEE Trans. Microw. Theory Tech. MTT-25 25 528-44

Twiss R Q 1958 Aust. J. Phys. 11 564-79

Vorgul I, Cairns R A and Bingham R 2005 Phys. Plasmas 12122903

Wu C S and Lee L C 1979 Astrophys. J. 230 621-6

Zarka P, Treumann R A, Ryabov B P and Ryabov V B 2001 Astrophys. Space Sci. 277 293-300 